\title{
I Plate-based Assay for Studying How Fungal Volatile Compounds (VCs) Affect Plant Growth and Development and the Identification of VCs via SPME-GC-MS Wenzhao Wang ${ }^{1, \#}$, Ningxiao $\mathrm{Li}^{2, \#}$, Xingzhong $\mathrm{Liu}^{1, *}$ and Seogchan $\mathrm{Kang}^{2, *}$
}

\author{
${ }^{1}$ State Key Laboratory of Mycology, Institute of Microbiology, Chinese Academy of Sciences, Beijing, \\ China; ${ }^{2}$ Department of Plant Pathology \& Environmental Microbiology, The Pennsylvania State \\ University, University Park, PA, USA \\ *For correspondence: liuxz@im.ac.cn; sxk55@psu.edu \\ \#Contributed equally to this work
}

\begin{abstract}
[Abstract] Biogenic volatile compounds (VCs) mediate various types of crucial intra- and inter-species interactions in plants, animals, and microorganisms owing to their ability to travel through air, liquid, and porous soils. To study how VCs produced by Verticillium dahliae, a soilborne fungal pathogen, affect plant growth and development, we slightly modified a method previously used to study the effect of bacterial VCs on plant growth. The method involves culturing microbial cells and plants in I plate to allow only VC-mediated interaction. The modified protocol is simple to set up and produces reproducible results, facilitating studies on this poorly explored form of plant-fungal interactions. We also optimized conditions for extracting and identifying fungal VCs using solid phase microextraction (SPME) coupled to gas chromatography-mass spectrometry (GC-MS).
\end{abstract}

Keywords: Arabidopsis thaliana, GC-MS, I plate, Plant-fungal interaction, SPME, Verticillium dahliae, Volatile compounds

[Background] Volatile compounds (VCs) have been shown or suggested to play varied and crucial roles in mediating organismal interactions within and across kingdoms. Plants rely on VCs to attract pollinators, seed dispersers, and parasitoids (Baldwin, 2010; Herrmann, 2010). Animals have evolved sophisticated olfactory systems to detect and respond to foods, threats, and mates through volatile cues (Buck, 2004). Similarly, microbial VCs seem to perform diverse functions such as suppressing competitors, regulating their population density, and controlling morphological transitions (Bailly and Weisskopf, 2012; Bennett et al., 2012; Bitas et al., 2013). Roles of microbial VCs in plant growth, development, and stress response have been investigated using several experimental setups that physically separate microbial cells from plants so that only VC-mediated interaction can occur (Ryu et al., 2003; Kai and Piechulla, 2009; Xie et al., 2009; Hung et al., 2013; Vaishnav et al., 2015). Among them, a method employing bipartite Petri plate, also called I plate, has been most frequently used. We adopted I plate to study the effect of VCs produced by soilborne fungal pathogens on plant growth, development, and responses to biotic and abiotic stresses (Bitas et al., 2015; Li and Kang, 2018; Li et al., 2018b). In addition, we optimized a scheme for VC extraction and analysis to help identify fungal VCs that are responsible for modulating plant growth and development (Li et al., 2018b).

Here, we provide a detailed protocol for setting up an I plate assay used for evaluating the effect of 
VCs produced by Verticillium dahliae, a devastating soilborne fungal pathogen that infects hundreds of plant species, on Arabidopsis thaliana. This protocol enables rapid and straightforward determination of if and how fungal VCs affect plants. We also describe a protocol for capturing VCs through solid phase microextraction (SPME) and analyzing extracted VCs via gas chromatography-mass spectrometry (GCMS). In combination, these protocols will help explore how VCs produced by diverse fungi affect plants and can also be applied to study VC-mediated interactions between microbes.

\section{Materials and Reagents}

1. Surgical blades \#10 and \#11

2. Parafilm (Bemis, catalog number: PM-99)

3. Paper towel

4. $10 \mu \mathrm{l}$ and $1,000 \mu \mathrm{l}$ micropipette tips

5. $100 \times 15 \mathrm{~mm}$ I plate (VWR, catalog number: 25384-310)

6. $100 \times 100 \mathrm{~mm}$ square plate (VWR, catalog number: 10799-140)

7. $100 \times 15 \mathrm{~mm}$ (VWR, catalog number: 25384-302) and $60 \times 15 \mathrm{~mm}$ (VWR, catalog number: 25384-092) Petri plates

8. $1.7 \mathrm{ml}$ microcentrifuge tube (VWR, catalog number: 87003-294)

9. $25 \mathrm{ml}$ serological pipette (VWR, catalog number: 89130-900)

10. Filter unit with $0.2 \mu \mathrm{M}$ cellulose membrane (Nalgene, catalog number: 121-0020)

11. $1.5 \mathrm{ml}$ sample vial (Shimadzu, catalog number: 221-34274-91), white cap with septum

12. V. dahliae strains PD322 and PD413 (conidial suspension in $20 \%$ glycerol and stored at $-80^{\circ} \mathrm{C}$ )

13. A. thaliana ecotype Col-0 seeds (Lehle Seed Co.)

14. Sterile MilliQ water

15. Murashige and Skoog (MS) basal medium (Sigma-Aldrich, catalog number: M0404-10L)

16. Sucrose (Alfa Aesar, catalog number: A15583)

17. Granulated agar (Difco, catalog number: 214530)

18. Potato dextrose agar (PDA) (Difco, catalog number: 213400)

19. 200 proof ethanol (KOPTEC, catalog number: 64-17-5)

20. $6 \%$ sodium hypochlorite (CLOROX)

21. n-Hexane (EMSURE, catalog number: 1043744000)

22. $\mathrm{C}_{7}-\mathrm{C}_{30}$ saturated alkanes (Sigma-Aldrich, catalog number: $49451-\mathrm{U}$ )

23. $99.99 \%$ pure helium gas

24. 0.5x PDA medium (see Recipes)

25. MS agar medium (see Recipes)

\section{Equipment}

1. Pyrex glass bottle 
2. $10 \mu \mathrm{l}$ and $1,000 \mu \mathrm{l}$ micropipettes

3. Scalpel

4. Forceps

5. Cork borer (5 $\mathrm{mm}$ in diameter)

6. SPME fiber holder (Supelco, catalog number: $57330-U$ )

7. $15 \mathrm{ml}$ clear glass vial (Supelco, catalog number: 27159), screw cap with PTFE/silicone septum

8. SPME fiber assembly with 50/30 $\mu \mathrm{m}$ DVB/CAR/PDMS fiber coating (Supelco, catalog number: 57328-U)

9. Electric pipette controller (Drummond Scientific Co., catalog number: 4-000-110-TC)

10. Vortex (VWR, model: Genie 2)

11. Table-top centrifuge (Eppendorf, model: $5417 \mathrm{C}$ )

12. Table-top shaker (VWR, catalog number: 57018-754)

13. Analytical balance (Mettler Toledo, model: AE-100)

14. Dissecting microscope (Zeiss, model: Stemi 2000-C)

15. Incubator (Sheldon Manufacturing, model: 1510E)

16. Plant growth chamber (Conviron, model: CMP5090)

17. Flexible-arm electrode holder (Mettler Toledo, catalog number: 30266628)

18. GC-MS system (Shimadzu, model: GCMS-QP2010 ultra) equipped with AOC-20i auto injector (Shimadzu, catalog number: 221-72315-48)

19. Rtx-Wax capillary ( $60 \mathrm{~m}, 0.25 \mathrm{~mm}$ ID and $0.25 \mu \mathrm{m}$ df) column (Restek, catalog number: 12426)

20. $4{ }^{\circ} \mathrm{C}$ refrigerator

21. Autoclave

\section{Software}

1. Image J (version 1.52a)

2. GC-MS Solution (Shimadzu, version 2.72), a package supporting GC-MS real-time and postrun analyses

3. National Institute of Standards and Technology (NIST) Mass spectral library (Shimadzu, version 11)

\section{Procedure}

A. I plate assay (Figure 1)

1. Use a sterile $10 \mu$ pipette tip to streak $V$. dahliae stock on $0.5 x$ PDA (Recipe 1) plate in a zigzag pattern and incubate at $22^{\circ} \mathrm{C}$ for 10 days.

2. Surface sterilize $A$. thaliana seeds as follows:

a. Add $1 \mathrm{ml} 95 \%$ ethanol to a $1.7 \mathrm{ml}$ microcentrifuge tube containing seeds, vortex, and incubate for $1 \mathrm{~min}$. 
b. After removing ethanol, wash once with sterile MilliQ water and discard the water.

c. Add $1 \mathrm{ml} 6 \%$ sodium hypochlorite solution, vortex, and incubate for $15 \mathrm{~min}$ by shaking at $100 \mathrm{rpm}$.

d. Wash twice with sterile MilliQ water after removing the sodium hypochlorite solution.

e. Incubate seeds in $1 \mathrm{ml}$ sterile MilliQ water at $4{ }^{\circ} \mathrm{C}$ in darkness for 3 days.

3. Prepare square plates with MS agar (Recipe 2) and slice the medium into $10 \times 10 \mathrm{~mm}$ pieces using a sterilized scalpel with blade \#10.

4. Hold one seed at the tip of a $10 \mu \mathrm{l}$ micropipette using suction and then release the seed onto each agar piece. Seal the plate with two layers of Parafilm and place in a plant growth chamber set at $22{ }^{\circ} \mathrm{C}, 12 \mathrm{~h}$ light $\left(4,500\right.$ lux, $60 \mu \mathrm{mol}$ photons $\left.\mathrm{m}^{-2} \mathrm{~s}^{-1}\right)$, and $60 \%$ relative humidity for 7 days.

5. Prepare I plate by adding $8 \mathrm{ml} \mathrm{MS}$ agar to one compartment and $8 \mathrm{ml} 0.5 \mathrm{x}$ PDA to the other compartment.

6. Transfer five A. thaliana seedlings (similar in size and growth stage) along with attached agar piece to the MS side of I plate using a sterilized scalpel with blade \#10.

7. Use a heat-sterilized cork borer to generate culture plugs along the actively growing margin of V. dahliae culture and place one plug (upside down) to the far end of the PDA side of I plate using a sterilized scalpel with blade \#11.

8. Seal the inoculated I plate with two layers of Parafilm and place in a plant growth chamber for a designated amount of time for each experiment (see Li et al., 2018b for specific examples).

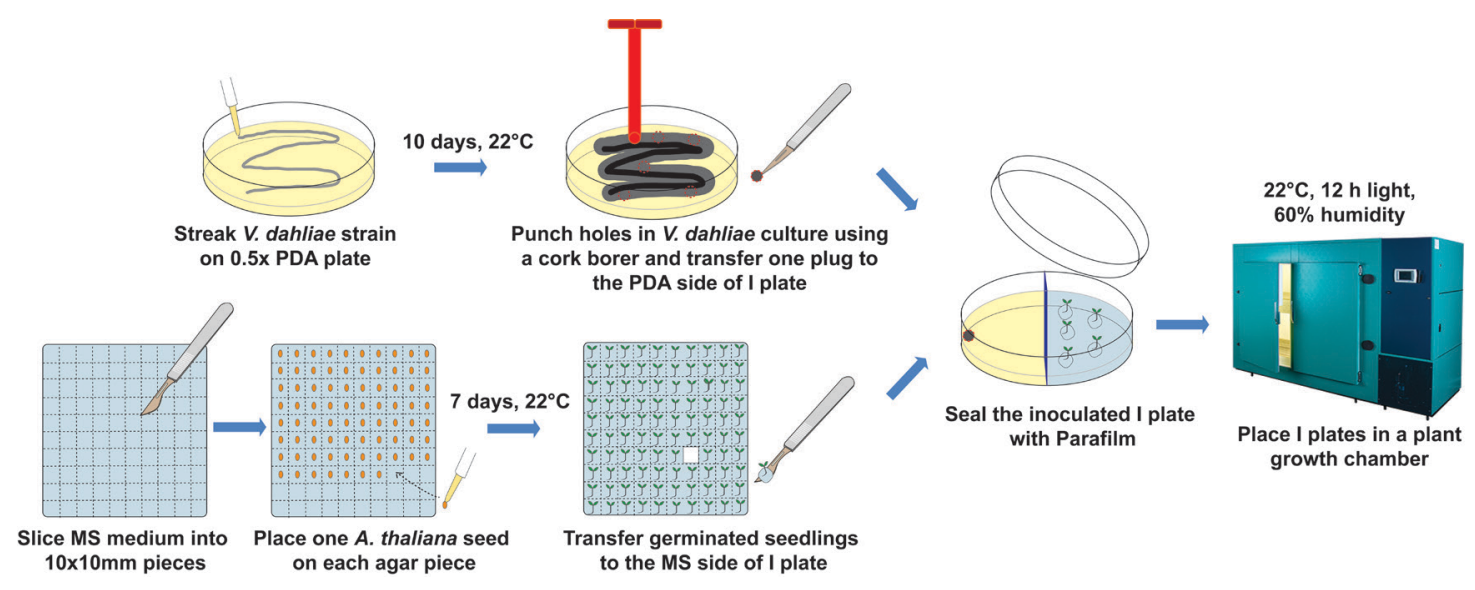

Figure 1. Workflow of the I plate-based assay

B. Extraction of VCs produced by $V$. dahliae (Figure 2)

1. Inoculate a plug of $V$. dahliae culture on $8 \mathrm{ml}$ PDA slant in a $15 \mathrm{ml}$ clear glass vial. Seal the vial with Parafilm.

2. Incubate at $22^{\circ} \mathrm{C}$ until the culture fully covers the surface of PDA slant, which typically takes 8 days.

3. Replace Parafilm with a screw cap containing PTFE/silicone septum and incubate for one day.

4. Condition the SPME fiber before VC extraction by placing the SPME needle into the GC injection 
port set at $230{ }^{\circ} \mathrm{C}$ for $1 \mathrm{~h}$.

5. Insert the conditioned SPME fiber in the injection port and starting the GC temperature program shown in Table 1. Desorb the fiber for $5 \mathrm{~min}$ in the injection port. Retract the fiber and remove the needle after $5 \mathrm{~min}$ and check resulting chromatograms when the program is completed. The intensity of background peaks (= blank sample) should be very low. Otherwise, repeat the desorption of the fiber.

6. Extract $\mathrm{VCs}$ for $1 \mathrm{~h}$ by leaving the SPME fiber in the headspace of sampling vial. Use a flexiblearm electrode holder to lock the position of the SPME fiber holder so that the insertion depth of fiber is uniform for all extractions.
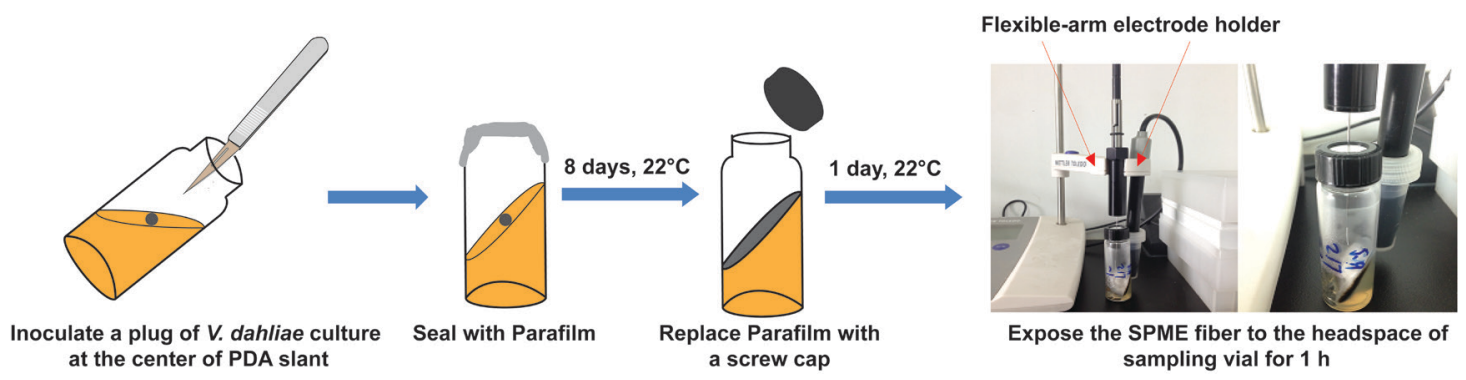

Figure 2. Workflow of volatile compound extraction

Table 1. Conditions for gas chromatography-mass spectrometry analysis

\begin{tabular}{lll}
\hline Column & Type & Rtx-Wax capillary column \\
& Length $(\mathrm{m})$ & 60 \\
& $\mathrm{ID}(\mathrm{mm})$ & 0.25 \\
& $\mathrm{D}_{\mathrm{f}}(\mu \mathrm{m})$ & 0.25 \\
\hline Carrier Gas & Gas type & Helium $(99.99 \%$ pure $)$ \\
& Total flow & $10 \mathrm{ml} \mathrm{min}^{-1}$ \\
& Flow rate & $1 \mathrm{ml} \mathrm{min}^{-1}$ \\
& Purge flow & $4 \mathrm{ml} \mathrm{min}^{-1}$ \\
& Pressure & $109.2 \mathrm{kPa}^{-}$ \\
\hline Inlet & Temperature & $230{ }^{\circ} \mathrm{C}$ \\
& Injection mode & $\mathrm{Splitless}$ \\
\hline Oven Temp. Program & Isothermal heating & $5 \mathrm{~min}^{\circ} 35^{\circ} \mathrm{C}$ \\
& Temperature gradient & $5{ }^{\circ} \mathrm{C} \mathrm{min}{ }^{-1}$ to $230{ }^{\circ} \mathrm{C}$ \\
& Final heating & $15 \mathrm{~min}^{\circ} 230{ }^{\circ} \mathrm{C}$ \\
\hline Mass Spectra & Mass scan method & Total-ion-count $(\mathrm{TIC})$ \\
& Mass scan range & 35 to $500 \mathrm{~m} / \mathrm{z}$ \\
& Run time & 1.5 to $59.0 \mathrm{~min}$ \\
& Event time & $0.3 \mathrm{~s}$ \\
\hline
\end{tabular}


C. GC-MS analysis of extracted VCs (Figure 3)

1. Analyze the extracted VCs using a GC-MS system (manual injection mode, Figure 3A) by following the conditions described in Table 1. Insert the SPME needle into the GC injection port immediately after retracting it from each sampling vial. Desorb VCs bound to the SPME fiber for 5 min. An Rtx-Wax capillary column is used as it exhibits better retention and separation of polar VCs.

2. Place the fiber in the $\mathrm{GC}$ injection port for $\mathbf{2 0} \mathrm{min}$ for desorption before extracting another sample of VCs.

3. Analyze $\mathrm{C}_{7}-\mathrm{C}_{30}$ saturated alkane standards using the automatic sample injection mode (Figure $3 B$ ). Connect the AOC-20i auto injector to the GC injection port. Place a $1.5 \mathrm{ml}$ sample vial containing $0.5 \mathrm{ml}$ solution of standards (1:50 dilution with $\mathrm{n}$-hexane) on the rack of the auto injector. Inject $1 \mu$ solution into the GC-MS system and analyze using the same column and conditions (Table 1).
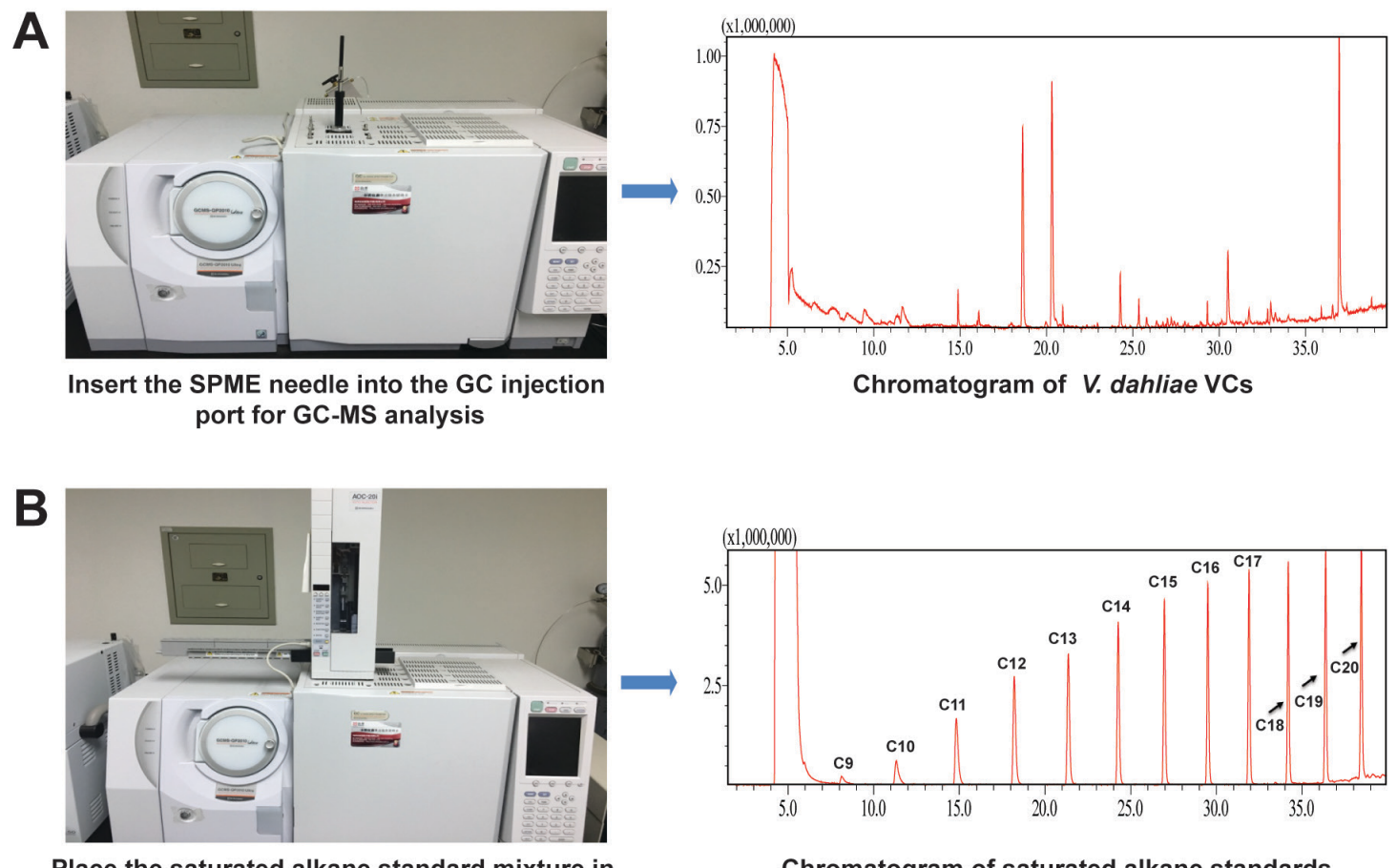

Place the saturated alkane standard mixture in the auto injector for GC-MS analysis

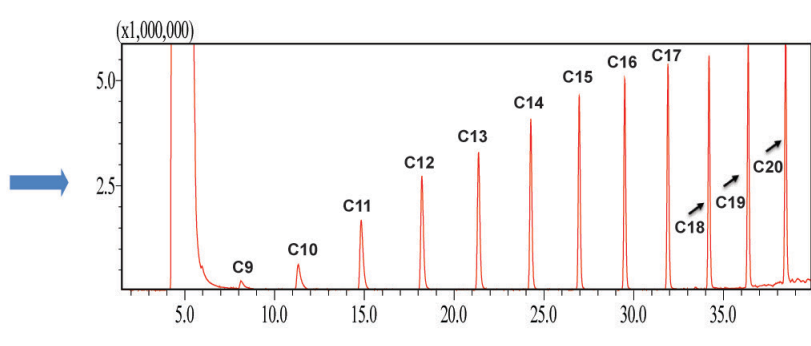

Chromatogram of saturated alkane standards

Figure 3. Gas chromatography-mass spectrometry analysis. A. Extracted volatile compounds (VCs) from $V$. dahliae were analyzed via the manual injection mode. B. Alkane standards were analyzed using the automatic sample injection mode.

\section{Data analysis}

A. Assessment of root growth and development after individual VC treatments

1. After co-cultivation, use forceps to gently pull $A$. thaliana seedlings from the medium without damaging their roots. 
2. Remove excess moisture on the roots using a paper towel.

3. Detaching roots from the shoot using forceps, weigh the roots immediately using an analytical balance.

4. Mount the roots on a flat surface (e.g., $100 \times 15 \mathrm{~mm}$ Petri plate) and add $3 \mathrm{ml}$ water on them.

5. Gently spread the roots using forceps so that the primary and lateral roots do not overlap.

6. Use the bottom of $60 \times 15 \mathrm{~mm}$ Petri plate to flatten the roots (Figure 4A) for subsequent measurements:

a. Count the number of lateral roots, including all branches, under a dissecting microscope.

b. Take pictures of the roots and import them to ImageJ. Follow the instruction in Image J to measure the primary root length (Figure 4B).

7. Calculate the lateral root density of each sample by dividing the number of lateral roots by the primary root length.

\section{A}

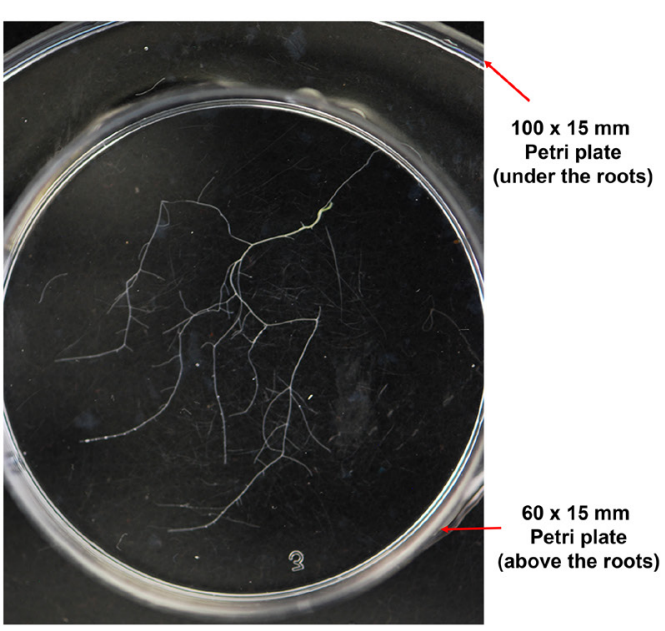

B

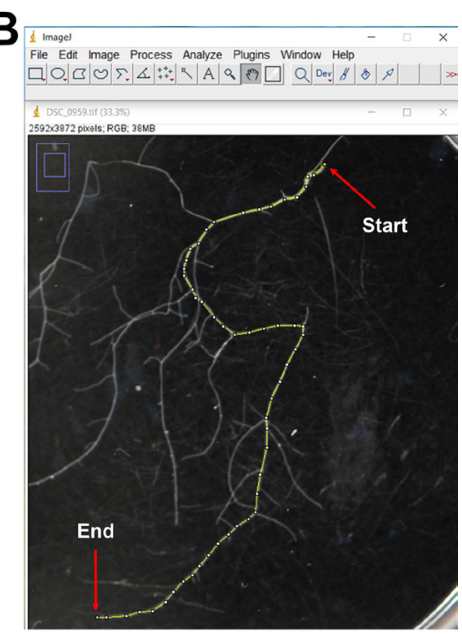

Figure 4. Analysis of $A$. thaliana roots after treating with volatile compounds produced by $V$. dahliae. A. Flattened $A$. thaliana roots by pressing with a $60 \times 15 \mathrm{~mm}$ Petri plate. B. Snapshot of ImageJ data used to measure the length of the primary root (indicated by the yellow line) after VC treatment. The start and end of the primary root were indicated using red arrows.

B. Identification of individual compounds

1. Peak finding, peak integration, and retention time correction were performed using the post-run analysis software in the GC-MS Solution package.

2. The putative identity of each compound (peak) was determined using the following methods.

a. Compare resulting mass spectral profiles with reference data archived in the NIST mass spectral library (version 11). The top hit(s) with match factors $\geq 90 \%$ were put on a "positive list" of tentatively identified compounds.

b. Calculate the retention index (RI) of each compound using the following equation (for temperature programmed chromatography) (Kováts, 1958). 


$$
\mathrm{RIx}=100\left[\frac{(T x-T n)}{(T N-T n)}+n\right]
$$

where,

$\mathrm{x}=$ Unknown compound in the sample

$\mathrm{n}=$ The number of carbons in the alkane preceding the unknown compound

$\mathrm{N}=$ The number of carbons in the alkane following the unknown compound

$\mathrm{Tx}=$ The retention time of the unknown compound

$\mathrm{Tn}=$ The retention time of the preceding alkane

$\mathrm{TN}=$ The retention time of the following alkane

The experimentally obtained RI of each peak was compared to those in the NIST Chemistry WebBook (https://webbook.nist.gov, using polar columns). For positive confirmation of identity, a maximum relative deviation of $\pm 2 \%$ from published values was used (Stoppacher et al., 2010).

\section{Notes}

1. The I plate assay may not be suitable for studying the effect of VCs produced by fungi that grow rapidly, as fungal mycelia may grow over the central divider of I plate and contaminate the MS medium. We removed agar strips from both sides of the divider of I plate to prevent this kind of contamination when we studied fungi that grow faster than V. dahliae (Bitas et al., 2015). However, for fungi like Trichoderma, this measure was not sufficient (Li et al., 2018a).

2. Due to the limited space of I plate, only small plants (e.g., A. thaliana and Nicotiana benthamiana) in their early growth stage are suitable for this assay.

3. Under the growth conditions used, growth promoting effect on $A$. thaliana becomes noticeable as early as after 7 days of co-cultivation with V. dahliae (Li et al., 2018b).

4. Because $A$. thaliana starts to initiate inflorescence development after 14 days of co-cultivation, we recommend that the duration of co-cultivation should not exceed 14 days.

5. Here, we only described how to analyze the root growth and development after VC exposure. Other traits of VC-exposed plants, such as shoot weight, chlorophyll content, physiological and molecular changes, can also be analyzed (e.g., Zhang et al., 2007 and 2008; Li et al., 2018b).

6. Several SPME fibers and GC columns may need to be evaluated to optimize the scheme for VC extraction and analysis. In our research, the DVB/CAR/PDMS 50/30 $\mu \mathrm{m}$ fiber coating extracted the largest number of VCs, and the Rtx-Wax capillary column enabled better separation of extracted VCs than the DB-5 column.

7. Analysis of VCs extracted from uninoculated PDA slant is necessary to exclude VCs derived from the medium and the environment. 


\section{Recipes}

1. $0.5 x$ PDA medium $(1 \mathrm{~L})$

$$
19.5 \mathrm{~g} \text { PDA }
$$

Pour the autoclaved medium after cooling it down to $50^{\circ} \mathrm{C}$

2. MS agar medium ( $1 \mathrm{~L})$

\section{$4.5 \mathrm{~g} \mathrm{MS}$ and $7.5 \mathrm{~g}$ agar}

Add $5 \mathrm{ml} 50 \%(\mathrm{w} / \mathrm{v})$ sucrose (filter sterilized) after cooling down the autoclaved medium to $50{ }^{\circ} \mathrm{C}$ Mix well before pouring

\section{Acknowledgments}

Our research on fungal VCs has been supported by a grant to SK from the USDA-Specialty Crop Multi-State Program (AM170200XXXXG006), a fellowship to NL from the Storkan-Hanes-McCaslin Foundation, and the Huck Dissertation Research Award to NL. The I plate assay and the method for VC extraction were adopted from Ryu et al. (2003) and Stoppacher et al. (2010), respectively.

\section{Competing interests}

The authors declare no conflicts of interest or competing interests.

\section{References}

1. Bailly, A. and Weisskopf, L. (2012). The modulating effect of bacterial volatiles on plant growth: current knowledge and future challenges. Plant Signal Behav 7(1): 79-85.

2. Baldwin, I. T. (2010). Plant volatiles. Curr Biol 20(9): R392-397.

3. Bennett, J. W., Hung, R., Lee, S. and Padhi, S. (2012). 18 Fungal and bacterial volatile organic compounds: An overview and their role as ecological signaling agents. In: Hock, B. (Ed.). Fungal Associations. Springer, Berlin, Heidelberg, 373-393.

4. Bitas, V., Kim, H. S., Bennett, J. W. and Kang, S. (2013). Sniffing on microbes: diverse roles of microbial volatile organic compounds in plant health. Mol Plant Microbe Interact 26(8): 835-843.

5. Bitas, V., McCartney, N., Li, N., Demers, J., Kim, J. E., Kim, H. S., Brown, K. M. and Kang, S. (2015). Fusarium oxysporum volatiles enhance plant growth via affecting auxin transport and signaling. Front Microbiol 6: 1248.

6. Buck, L. B. (2004). Olfactory receptors and odor coding in mammals. Nutr Rev 62(11 Pt 2): S184-188; discussion S224-141.

7. Herrmann, A. (2010). The chemistry and biology of volatiles. In: Herrmann, A. (Ed.). Chichester, John Wiley \& Sons, Ltd.

8. Hung, R., Lee, S. and Bennett, J. W. (2013). Arabidopsis thaliana as a model system for testing 
the effect of Trichoderma volatile organic compounds. Fungal Ecology 6(1): 19-26.

9. Kai, M. and Piechulla, B. (2009). Plant growth promotion due to rhizobacterial volatiles--an effect of $\mathrm{CO}_{2}$ ? FEBS Lett 583(21): 3473-3477.

10. Kováts, E. (1958). Gas-chromatographische charakterisierung organischer verbindungen. Teil 1: Retentionsindices aliphatischer halogenide, alkohole, aldehyde und ketone. Helvetica Chimica Acta 41(7): 1915-1932.

11. Li, N., Alfiky, A., Wang, W., Islam, M., Nourollahi, K., Liu, X. and Kang, S. (2018a). Volatile compound-mediated recognition and inhibition between Trichoderma biocontrol agents and Fusarium oxysporum. Front Microbiol 9: 2614.

12. Li, N. and Kang, S. (2018). Do volatile compounds produced by Fusarium oxysporum and Verticillium dahliae affect stress tolerance in plants? Mycology 9(3): 166-175.

13. Li, N., Wang, W., Bitas, V., Subbarao, K., Liu, X. and Kang, S. (2018b). Volatile compounds emitted by diverse Verticillium species enhance plant growth by manipulating auxin signaling. Mol Plant Microbe Interact 31(10): 1021-1031.

14. Ryu, C. M., Farag, M. A., Hu, C. H., Reddy, M. S., Wei, H. X., Pare, P. W. and Kloepper, J. W. (2003). Bacterial volatiles promote growth in Arabidopsis. Proc Natl Acad Sci U S A 100(8): 4927-4932.

15. Stoppacher, N., Kluger, B., Zeilinger, S., Krska, R. and Schuhmacher, R. (2010). Identification and profiling of volatile metabolites of the biocontrol fungus Trichoderma atroviride by HSSPME-GC-MS. J Microbiol Methods 81(2): 187-193.

16. Vaishnav, A., Kumari, S., Jain, S., Varma, A. and Choudhary, D. K. (2015). Putative bacterial volatile-mediated growth in soybean (Glycine max L. Merrill) and expression of induced proteins under salt stress. J Appl Microbiol 119(2): 539-551.

17. Xie, X., Zhang, H. and Pare, P. W. (2009). Sustained growth promotion in Arabidopsis with longterm exposure to the beneficial soil bacterium Bacillus subtilis (GB03). Plant Signal Behav 4(10): 948-953.

18. Zhang, H., Kim, M. S., Krishnamachari, V., Payton, P., Sun, Y., Grimson, M., Farag, M. A., Ryu, C. M., Allen, R., Melo, I. S. and Pare, P. W. (2007). Rhizobacterial volatile emissions regulate auxin homeostasis and cell expansion in Arabidopsis. Planta 226(4): 839-851.

19. Zhang, H., Xie, X., Kim, M. S., Kornyeyev, D. A., Holaday, S. and Pare, P. W. (2008). Soil bacteria augment Arabidopsis photosynthesis by decreasing glucose sensing and abscisic acid levels in planta. Plant J 56(2): 264-273. 